\title{
On Odd Perfect, Quasiperfect, and Odd Almost Perfect Numbers
}

\section{By Masao Kishore}

\begin{abstract}
We establish upper bounds for the six smallest prime factors of odd perfect, quasiperfect, and odd almost perfect numbers.
\end{abstract}

1. Suppose $N=\prod_{i=1}^{r} p_{i}^{a_{i}}$ is an odd perfect (OP) number, i.e. $\sigma(N)=2 N$, where $p_{i}$ 's are odd primes, $p_{1}<\cdots<p_{r}$, and $a_{i}$ 's are positive integers. Grun [1] proved that

$$
p_{1}<2+2 r / 3
$$

and Pomerance [5] proved that

$$
p_{i}<(4 r)^{2^{i(i+1) / 2}} \text { for } 1<i<r .
$$

In [3] we showed that if $N$ is an odd integer and the number $\omega(N)$ of distinct prime factors of $N$ is 5 , then

$$
|2-\sigma(N) / N|>10^{-14} \text {. }
$$

From this it follows immediately that if $M$ is an odd integer, $\sigma(M)=2 M+L$, and if $|L / M|<10^{-14}$, then $\omega(M)>6$. OP, quasiperfect (QP) numbers, i.e. $\sigma(N)=2 N$ +1 , and odd almost perfect (OAP) numbers, i.e. $\sigma(N)=2 N-1$, are such examples.

Also, it can be proved from (2) that if $M=\prod_{i=1}^{r} p_{i}^{a_{i}}$ is OP,

$$
p_{6}<2 \cdot 10^{14}(r-5) \text {. }
$$

However, if we consider only those $N=\Pi_{i-1}^{5} p_{i}^{a_{1}}$ in (2) for which $\Pi_{i=1}^{r} p_{i}^{a_{4}}$ is OP, then exponents $a_{i}$ are restricted, and hence we have a better lower bound in (2). Consequently we have a better upper bound for $p_{6}$.

In this paper we prove

Theorem. Suppose $M=\prod_{i=1}^{r} p_{i}^{a_{i}}$. If $M$ is $O P$ or $Q P$,

$$
p_{i}<2^{2^{i-1}}(r-i+1) \text { for } 2<i<6 \text {. }
$$

If $M$ is $O A P$,

$$
\begin{aligned}
& p_{i}<2^{2^{i-1}}(r-i+1) \text { for } 2<i<5, \text { and } \\
& p_{6}<23775427335(r-5) .
\end{aligned}
$$

Although our Theorem gives upper bounds for $p_{i}$ only for $2<i<6$, they are better than (1). For example, if $M$ is OP, then $p_{5}<65536(r-4)$ by our Theorem

Received January 21, 1980; revised July 28, 1980.

1980 Mathematics Subject Classification. Primary 10A20. 
and $p_{r}>100110$ by Hargis and McDaniel [2]. Hence, we have another proof that $\omega(M)>6$.

2. In order to prove our Theorem, we need three lemmas.

Definition. $S(N)=\sigma(N) / N$.

Lemma 1. Suppose $M=\Pi_{i=1}^{r} p_{i}^{a_{i}}$ is $O P$. Then

$$
S\left(\prod_{i=1}^{5} p_{i}^{a_{i}}\right)<\frac{3}{2} \frac{5}{4} \frac{17}{16} \frac{257}{256} \frac{65537}{65536}=\alpha \approx 2-4 / 10^{10} .
$$

Proof. Since $M$ is OP, by Euler,

$$
\text { if } p_{i} \equiv 1(4), \quad a_{i} \equiv 0,1,2(4), \quad \text { and if } \quad p_{i} \equiv 3(4), \quad a_{i} \equiv 0 \text { (2), }
$$

and if $q$ is an odd prime factor of $\sigma\left(p_{i}^{a_{i}}\right)$ for some $i$, then $q \mid M$. Suppose

$$
\alpha<S\left(\prod_{i=1}^{5} p_{i}^{a_{4}}\right)<2
$$

and $q \neq p_{i}$ for $1<i<5$. If $q<10^{9}$, then

$$
\begin{aligned}
\log 2 & =\log S(M)>\log S\left(\prod_{i=1}^{5} p_{i}^{a_{i}}\right)+\sum_{i=6}^{r} \log S\left(p_{i}^{a_{i}}\right) \\
& >\log \alpha+\log (q+1) / q>\log \alpha+\log \left(10^{9}+1\right) / 10^{9}>\log 2,
\end{aligned}
$$

a contradiction. Hence,

$$
\begin{aligned}
& \text { If } q \text { is an odd prime factor of } \sigma\left(p_{i}^{a^{q}}\right) \text { for } \\
& \text { some } i \text { and } q \neq p_{j} \text { for } 1<j<5 \text {, then } q>10^{9} \text {. }
\end{aligned}
$$

As in [3], we used a computer (PDP11 at the University of Toledo) to find odd integers $\Pi_{i=1}^{5} p_{i}^{a_{i}}$ satifying (3) and (4). There were infinitely many such $\Pi_{i=1}^{5} p_{i}^{a_{1}}$. (However, there were finitely many (just over one hundred) $\Pi_{i=1}^{5} p_{i}^{a_{1}}$ if $a_{i}<a\left(p_{i}\right)$ where

$$
a\left(p_{i}\right)=\min \left\{a_{i} \mid a_{i} \text { satisfies (3) and } p_{i}^{a_{1+1}}>10^{11}\right\} .
$$

See [3].) In every case such $\Pi_{i=1}^{5} p_{i}^{a_{i}}$ had a component $p_{i}^{a_{i}}$ such that $a_{i}<a\left(p_{i}\right), q$ is an odd prime factor of $\sigma\left(p_{i}^{a_{i}}\right), q \neq p_{j}$ for $1<j<5$ and $q<10^{9}$, contradicting (5). Q.E.D.

Lemma 2. Suppose $M=\Pi_{i=1}^{r} p_{i}^{a_{i}}$ is $Q P$. Then

$$
S\left(\prod_{i=1}^{5} p_{i}^{a_{i}}\right)<\frac{3}{2} \frac{5}{4} \frac{17}{16} \frac{257}{256} \frac{65537}{65536}=\alpha \approx 2-4 / 10^{10} .
$$

Proof. Since $M$ is QP, by [3], $r>6, S\left(\Pi_{i=1}^{5} p_{i}^{a_{1}}\right)<2$, and

$$
\begin{aligned}
& a_{i} \equiv 0(2) \text { for any } i, \\
& \text { if } p_{i}=3, a_{i}=4,12 \text { or }>24, \\
& \text { if } p_{i}=5, a_{i}=6 \text { or }>16, \\
& \text { if } p_{i}=17, a_{i}=2 \text { or }>8 .
\end{aligned}
$$


We used the computer to find odd integers $\Pi_{i=1}^{5} p_{i}^{a_{1}}$ satisfying (6) and

$$
\alpha<S\left(\prod_{i=1}^{5} p_{i}^{a_{i}}\right)<2,
$$

but there were none. Q.E.D.

Lemma 3. Suppose $M=\Pi_{i=1}^{r} p_{i}^{a_{i}}$ is OAP. Then

$$
S\left(\prod_{i=1}^{5} p_{i}^{a_{i}}\right)<S\left(3^{12}\right) \frac{5}{4} S\left(17^{6}\right) \frac{257}{256} \frac{62939}{62938}=\beta \approx 2-8 / 10^{11}
$$

Proof. Since $M$ is OAP, by [3], $r \geqslant 6$ and

$$
\begin{aligned}
& a_{i} \equiv 0(2) \text { for all } i, \\
& \text { if } p_{i}=3, a_{i}=12,16 \text { or } \geqslant 24, \\
& \text { if } p_{i}=5, a_{i}=2,10 \text { or }>16, \\
& \text { if } p_{i}=257, a_{i}>16 .
\end{aligned}
$$

We used the computer to find odd integers $\Pi_{i=1}^{5} p_{i}^{a_{1}}$ satisfying (7) and

$$
\alpha<S\left(\prod_{i=1}^{5} p_{i}^{a_{i}}\right)<2
$$

and the results were

$$
\begin{array}{ll}
3^{a_{1} 5^{10} 17^{a_{3}} 257^{a_{4}} 65449^{a_{5}},}, & \text { where } a_{1}>24, a_{3}>8, a_{4}>16, a_{5}>2, \text { and } \\
3^{12} 5^{a_{2}} 17^{6} 257^{a_{4}} 62939^{a_{5}}, & \text { where } a_{2}>16, a_{4}>16, a_{5}>2 .
\end{array}
$$

Since

$$
\frac{3}{2} S\left(5^{10}\right) \frac{17}{16} \frac{257}{256} \frac{65449}{65448}<S\left(3^{12}\right) \frac{5}{4} S\left(17^{6}\right) \frac{257}{256} \frac{62939}{62938}=\beta,
$$

Lemma 3 follows. Q.E.D.

Proof of Theorem. We prove only the case $i=5$. Suppose $M=\Pi_{i=1}^{r} p_{i}^{a_{i}}$ is OP or $\mathrm{QP}, N=\Pi_{i=1}^{5} p_{i}^{a_{i}}$, and

$$
\frac{2}{2-\alpha}(r-5)+1 \leqslant p_{6}<\cdots<p_{r} .
$$

Since $\log (1+x)<x$ and $\log (1-x)<-x$ if $0<x<1$, we have, by Lemmas 1 and 2 ,

$$
\begin{aligned}
\log 2 & <\log S(M)=\log S(N)+\sum_{i=6}^{r} \log S\left(p_{i}^{a_{1}}\right) \\
& <\log \alpha+(r-5) \log S\left(p_{6}^{a_{6}}\right) \\
& <\log 2+\log \alpha / 2+(r-5) \log p_{6} /\left(p_{6}-1\right) \\
& =\log 2+\log (1-(2-\alpha) / 2)+(r-5) \log \left(1+1 /\left(p_{6}-1\right)\right) \\
& <\log 2-(2-\alpha) / 2+(r-5) /\left(p_{6}-1\right) \\
& <\log 2-(2-\alpha) / 2+(2-\alpha) / 2=\log 2,
\end{aligned}
$$

a contradiction. Hence,

$$
p_{6}<\frac{2}{2-\alpha}(r-5)+1=2^{2^{5}}(r-5)+1 .
$$

Since $p_{6}$ is a prime, $p_{6}<2^{2^{5}}(r-5)$. 
Suppose $M=\Pi_{i=1}^{r} p_{i}^{a_{i}}$ is OAP, $N=\Pi_{i=1}^{5} p_{i}^{a_{i}}$, and

$$
\frac{2}{2-\beta}(r-5)+1 \leqslant p_{6}<\cdots<p_{r}
$$

Since $M>10^{30}$ by [4] and $\log (1-x)<-x-x^{2} / 2$ if $0<x<1$, we have, by Lemma 3,

$$
\begin{aligned}
\log 2-\frac{1}{2} \cdot 10^{30} & \approx \log 2+\log \left(1-\frac{1}{2} \cdot 10^{30}\right) \\
& =\log \left(2-1 / 10^{30}\right)<\log (2-1 / M)=\log (S(M) / M) \\
& =\log S(N)+\sum_{i=6}^{r} \log S\left(p_{i}^{a_{i}}\right)<\log \beta+(r-5) \log p_{6} /\left(p_{6}-1\right) \\
& <\log 2+\log (1-(2-\beta) / 2)+(r-5) /\left(p_{6}-1\right) \\
& <\log 2-(2-\beta) / 2-(2-\beta)^{2} / 8+(2-\beta) / 2 \\
& =\log 2-(2-\beta)^{2} / 8 \approx \log 2-9 \cdot 10^{-22},
\end{aligned}
$$

a contradiction. Hence

$$
p_{6}<\frac{2}{2-\beta}(r-5)+1<23775427335(r-5)+1 \text {. }
$$

Since $p_{6}$ is a prime, $p_{6}<23775427335(r-5)$. Q.E.D.

Finally, we (re)state the following

TheOREM. Suppose $N=\Pi_{i=1}^{r} p_{i}^{a_{i}}$ is an integer.

(a) If $r=5,|2-S(N)|>2-S\left(3^{7} 5^{6} 17^{2} 233\right) \cdot 36550429 / 36550428>10^{-14}$.

(b) If $r=4,|2-S(N)|>2-S\left(3^{7} 5^{6} 17^{2} 233\right)>5 / 10^{8}$.

(c) If $r=3,|2-S(N)|>S\left(3^{5} 5^{2} 13\right)-2>3 / 10^{4}$.

(d) If $r=2,|2-S(N)|>2-\frac{3}{2} \frac{5}{4}=0.125$.

(e) If $r=1,|2-S(N)|>2-\frac{3}{2}=0.5$.

Mathematics Department

University of Toledo

Toledo, Ohio 43606

1. O. GrUN, “Über ungerade vollkommene Zahlen,” Math. Z., v. 55, 1952, pp. 353-354.

2. P. Hagis, JR. \& W. L. MCDANIEL, "On the largest prime divisor of an odd perfect number. II," Math. Comp., v. 29, 1975, pp. 922-924.

3. M. KISHORE, "Odd integers $N$ with five distinct prime factors for which $2-10^{12}<\sigma(N) / N<2$ + 10-12," Math. Comp., v. 32, 1978, pp. 303-309.

4. M. KISHORE, The Number of Distinct Prime Factors of $N$ for Which $\sigma(N)=2 N, \sigma(N)=2 N \pm 1$, and $\phi(N) \mid N-1$, Doctoral dissertation, Princeton University, Princeton, N. J., 1977.

5. C. Pomernnce, "Multiply perfect numbers, Mersenne primes, and effective computability," Math. Ann., v. 266, 1977, pp. 195-206. 